INPLASY

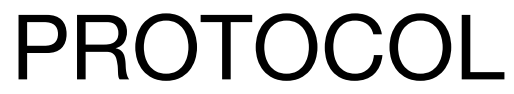

To cite: Chen et al.

Comparative efficacy and tolerance of intralymphatic, subcutaneous and sublingual immunotherapy for polleninduced allergic rhinitis: a network meta-analysis. Inplasy protocol 202210078. doi: 10.37766/inplasy2022.1.0078

Received: 16 January 2022

Published: 16 January 2022

Corresponding author:

Guoqi Sima

smgq73@163.com

Author Affiliation:

The first hospital of Jiaxing.

Support: None.

Review Stage at time of this submission: Preliminary searches.

Conflicts of interest:

None declared.

\section{Comparative efficacy and tolerance of intralymphatic, subcutaneous and sublingual immunotherapy for pollen- induced allergic rhinitis: a network meta-analysis}

Chen, R ${ }^{1}$ Sun, Y2; Sima, G³.

Review question / Objective: What is the effect of intralymphatic, subcutaneous and sublingual immunotherapy for pollen-induced allergic rhinitis.

Condition being studied: Immunotherapy is the classic treatment for allergic rhinitis. Intralymphatic immunotherapy is a new type of treatment. Currently, no studies have compared subcutaneous, sublingual and intralymphatic sublingual immunotherapy. At present, there is no review to compare the efficacy of intralymphatic, subcutaneous and sublingual immunotherapy for pollen-induced allergic rhinitis.

INPLASY registration number: This protocol was registered with the International Platform of Registered Systematic Review and Meta-Analysis Protocols (INPLASY) on 16 January 2022 and was last updated on 16 January 2022 (registration number INPLASY202210078).

\section{INTRODUCTION}

Review question / Objective: What is the effect of intralymphatic, subcutaneous and sublingual immunotherapy for polleninduced allergic rhinitis.
Condition being studied: Immunotherapy is the classic treatment for allergic rhinitis. Intralymphatic immunotherapy is a new type of treatment. Currently, no studies have compared subcutaneous, sublingual and intralymphatic sublingual immunotherapy. At present, there is no review to compare the efficacy of 
intralymphatic, subcutaneous and sublingual immunotherapy for polleninduced allergic rhinitis.

\section{METHODS}

Search strategy: Pubmed \#1 Rhinitis, Allergic (MeSH) \#2 Rhinitis, Allergic, Seasonal (MeSH) \#3 Hypersensitivity (MeSH) \#4 rhiniti* [Title/Abstract] OR allerg*[Title/ Abstract] OR hypersensitiv*[Title/Abstract] \#5 (\#1 OR \#2 OR \#3 OR \#4) \#6 Injections, Intralymphatic[Mesh] \#7 intralymph*[Title/Abstract] \#8 "Injections, Subcutaneous"[Mesh] \#9 subcutane*[Title/Abstract] \#10 "Sublingual Immunotherapy"[Mesh] \#11 sublingual ${ }^{\star}[$ Title/Abstract] \#12 \#6 OR \#7 OR \#8 OR \#9 OR \#10 OR \#11 \#13 pollen[Mesh]

\#14 pollen* [Title/Abstract]

\#15 \#13 OR \#14

\#16 \#5 AND \#12 AND \#15

The Cochrane Library

\#1 MeSH descriptor: [Rhinitis, Allergic] explode all trees

\#2 MeSH descriptor: [Rhinitis, Allergic, Seasonal] explode all trees

\#3 MeSH descriptor: [Hypersensitivity] explode all trees

\#4 (rhiniti $\left.{ }^{\star}\right): t i, a b, k w$ OR (allerg*):ti,ab,kw OR (hypersensitiv*):ti,ab,kw

\#5 \#1 OR \#2 OR \#3 OR \#4

\#6 MeSH descriptor: [Injections, Intralymphatic] explode all trees

\#7 (intralymph*):ti,ab,kw

\# 8 MeSH descriptor: [Injections, Subcutaneous] explode all trees

\#9 (subcutane $\left.{ }^{\star}\right)$ :ti,ab,kw

\#10 MeSH descriptor: [Sublingual Immunotherapy] explode all trees

\#11 (sublingual*):ti,ab,kw

\#12 \#6 OR \#7 OR \#8 OR \#9 OR \#10 OR \#11 \#13 MeSH descriptor: [Pollen] explode all trees

\#14 (pollen*):ti,ab,kw

\#15 \#13 OR \#14

\#16 \#5 AND \#12 AND \#15

Embase

\#1 'allergic rhinitis'/exp

\#2 'hypersensitivity'/exp

\#3 rhiniti*:ab,ti OR allerg*:ab,ti OR hypersensitiv*:ab,ti

\#4 \#1 OR \#2 OR \#3

\#5 intralymph*:ab,ti OR subcutane*:ab,ti OR sublingual*:ab,ti

\#6 'subcutaneous immunotherapy'/exp

\#7 'sublingual immunotherapy'/exp

\#8 \#5 OR \#6 OR \#7

\#9 'pollen'/exp

$\# 10$ pollen*:ab,ti

\#11 \#9 OR \#10

\#12 \#4 AND \#8 AND \#11

\#13 'crossover procedure':de OR 'doubleblind procedure':de OR 'randomized controlled trial': de OR 'single-blind procedure':de OR random*:de,ab,ti OR factorial*:de,ab,ti OR crossover*:de,ab,ti OR ((cross NEXT/1 over*):de,ab,ti) OR placebo*:de,ab,ti OR ((doubl* NEAR/1 blind $\left.^{*}\right):$ de,ab,ti) OR ((singl* NEAR/1

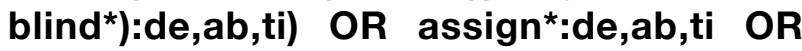
allocat $^{*}$ :de,ab,ti OR volunteer*:de,ab,ti \#14 \#12 AND \#13.

Participant or population: Patients diagnosed as pollen-induced allergic rhinitis

Intervention: Patients diagnosed as polleninduced allergic rhinitis were treated with intralymphatic, subcutaneous or sublingual immunotherapy.

Comparator: Control group: Patients diagnosed as pollen-induced allergic rhinitis were treated with placebo.

Study designs to be included: Randomized controlled trial.

Eligibility criteria: 1) Randomized controlled trial. 2) The patient was diagnosed with pollen-induced allergic rhinitis. 3) Patients were treated with SLIT, SCIT or ILIT. 4) Control group received Placebo.

Information sources: The main databases include Pubmed, Embase and the Cochrane Library. Other data sources include clinicaltrials. gov, the WHO ClinicalTrials registry, etc.

Main outcome(s): The main outcomes included symptom score and medication score. 
Additional outcome(s): Adverse events.

Quality assessment / Risk of bias analysis: Two authors independently evaluated the quality of studies. The Cochrane Collaboration's tool was used to assess the risks of bias and quality of RCTs. The following biases were investigated: random sequence generation, allocation concealment, blinding of participants and employees, blinding of result evaluation, incomplete outcome data, selective reporting, and others. When the methods were completely disclosed, there was a "low risk" of bias, a "high risk" when the methods were not mentioned, and an "unknown risk" when the methods were acknowledged but insufficiently comprehensive.

Strategy of data synthesis: Data were extracted by a self-made data extraction table, including the first author, year of publication, sample size, age, intervention measures, controlled measures, follow-up, allergen etc. Meta-analysis was performed by using $\mathbf{R}$ software. Dichotomous data were analyzed and reported as risk ratio (RR) and $95 \%$ confidence interval (Cl). Continuous data were presented as mean difference (MD) or standardized mean difference (SMD), standard deviation (SD), and $95 \% \mathrm{Cl}$. The standard error, median, range, and $95 \% \mathrm{Cl}$ were interpreted if the SD was not provided or could not be calculated. Discrepancies in the treatment effects among the trials were evaluated using heterogeneity $\left(I^{2}\right)$ statistics and deviance information criterion(DIC).

Subgroup analysis: According to the extracted data information, the possible differentiated subgroups were analyzed: subgroup analyses by the follow-up time.

Sensitivity analysis: If the number of articles included was greater than 10, sensitivity analysis was performed.

Language: Only English literature will be included.

Country(ies) involved: China.
Keywords: Allergic rhinitis; Intralymphatic immunotherapy; Subcutaneous i m munotherapy; Sublingual immunotherapy; Network meta-analysis.

Contributions of each author:

Author 1 - Ru Chen - Participate in literature screening and article writing.

Email: 753131058@qq.com

Author 2 - Yao Sun - Participate in literature screening and risk bias assessment.

Email: 1481050138@qq.com

Author 3 - Guoqi Sima - Corresponding author, participating in technical guidance.

Email: smgq73@163.com 